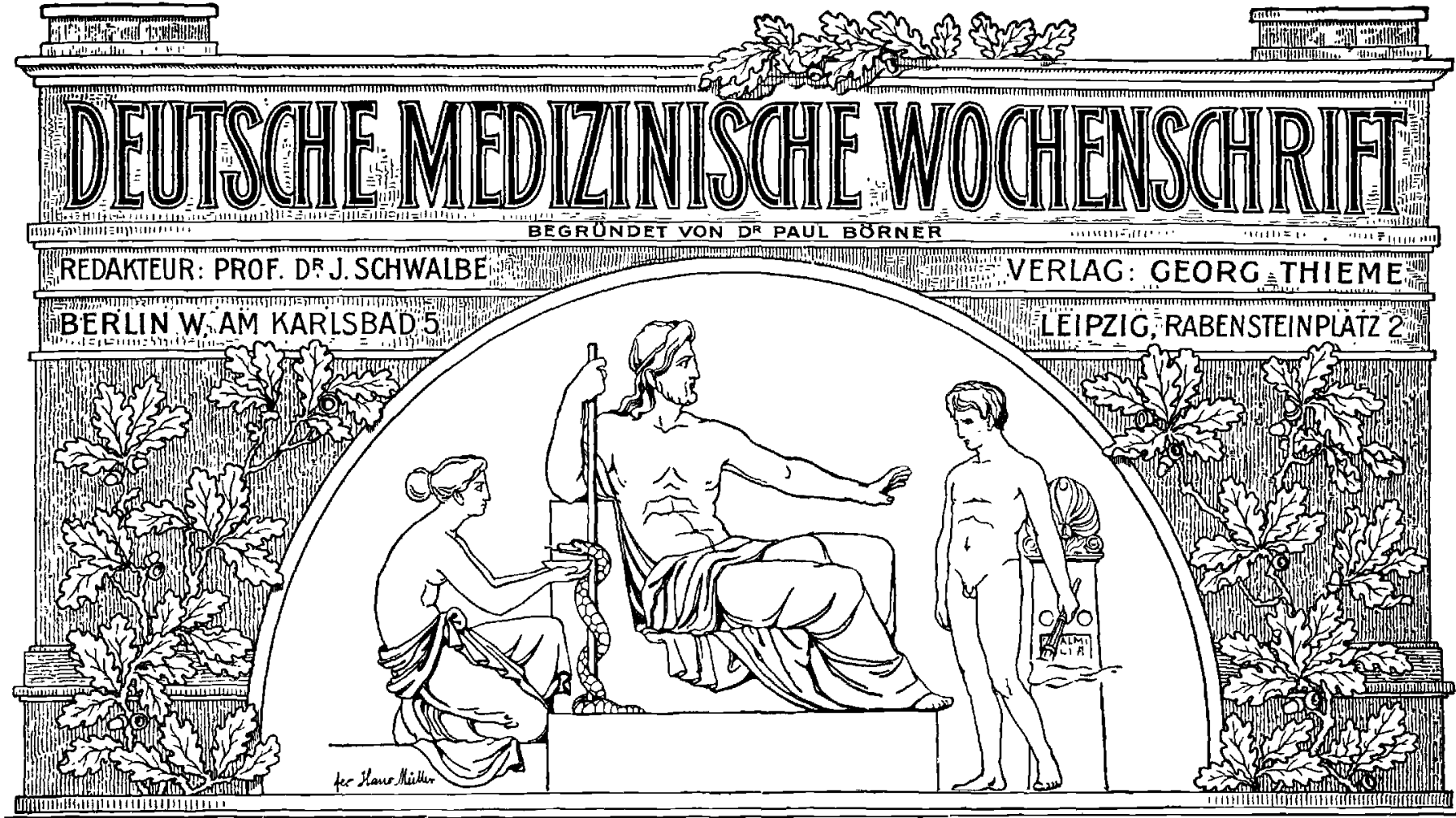

No. 49 .

Berlin, den 5. Dezember 1907.

33. Jahrgang.

Aus dem Pathologisch-bakteriologischen Institut des Neuen städtischen Krankenhauses in Karlsruhe.

\section{Neuere Forschungen über Morphologie und Entstehung der Geschwïlste.)}

\section{Von Prof. Dr. Ernst Schwalbe.}

Die "Entstehung der Geschwülste" ist in den letzten Jahren eine im Vordergrund des ärztlichen Interesses stehende Frage geworden. Scheint sich doch zum erstenmal ein Weg aufzutun, der zum Ziel zu führen verspricht, winkt doch, wenn auch in weiter Ferne, ein Licht, das uns die endlich ersehnte Aufklärung in Aussicht stellt. Kein Wunder, daß Hunderte von Forschern aller Kulturvölker heute sich mit den Geschwülsten beschäftigen, daß die verschiedensten Methoden versucht werden, um unser Wissen über die Geschwülste zu fördern. Eigene Institute, "Krebsinstitute“, in denen hervorragende Forscher tätig sind, haben sich das genannte Ziel als einzige Aufgabe gestellt; in Baden haben wir durch Czernys Energie in Heidelberg ein solches Institut erhalten, dessen wissenschaftliche Leitung in bewährtesten Händen liegt. Neu gegründete Prosekturen müssen nach ihrem Können und Vermögen an der großen Aufgabe mitarbeiton. Nur durch ruhigen, besonnenen Weiterbau auf den gegebenen Grundlagen der pathologischen Anatomie, vergleichenden und experimentellen Pathologie, sowie Immunitätsforschung können wir die ersehnte Förderung erhoffen. Freilich hat es immer einige Forscher gegeben und wird weiter solche geben, die sich über alles, was vor ihnen gearbeitet ist, kühn hinwegsetzen, die vorhandenen Grundlagen als ungeeignet zu jedem Fortschritt gänzlich verwerfen und auf einmal eine Theorie, meist vëllig fertig auf ungeahnten Voraussetzungen und angeblichen Entdeckungen errıchten. Bis jetzt hatten diese Entdecker aber auf Flugsand gebaut, ihr luftiges Hypothesengebäude wurde vom Winde verweht!

Namentlich hat die Ueberzeugung, ein Parasit müfte der Erreger der Geschwülste, insbesondere des Krebses, sein, auch bessere Forscher verführt, vorzeitig schon an die glückliche Lösung des Problems der Krebsentstehung zu glauben.

Die Lehre von den Geschwülsten ist in erster Linie eine morphologische Wissenschaft, und der Fortschritt in der Erkenntnis hat sich ebenso vollzogen wie in der Morphologie

1) Nach elnem im Karlsruher Aerzteverein gehaitenen Vortrag. überhaupt. Das, was wir mit bloßem Auge, makroskopisch, an den Geschwülsten wahrnehmen oder durch unsere übrigen Sinne feststellen können, ist nicht genügend, um eine tiefere Erkenntnis zu ermöglichen. Man hatte wohl auf diesem. Wege am Anfang des 19. Jahrhunderts einige Feststellungen erheben können, die auch jetzt noch keineswegs wertlos sind. Man unterschied die härteren von den weichen Geschwülsten, man hatte einer Gruppe wegen ihres fleischähnlichen Aussehens den Namen Sarkome gegeben, der noch heute gebräuchlich ist, wenn wir auch ganz andere Begriffe heutzutage mit dem alten Namen verbinden. - Die Vertiefung der Erkenntnis geschah durch das Mikroskop. J ohannes Müller gebührt das Verdienst, bedeutungsvolle Feststellungen über die Geschwülste zuerst mit Hilfe des Mikroskops gemacht zu haben, Rudolf Virchow legte den dauernden Grund für die heute gewonnene Kenntnis des Aufbaues der Geschwülste mit Hilfe des Mikroskops.

Wie die Anatomie der Anwendung des verbesserten Mikro. skops im 19. Jahrhundert nicht nur die Histologie, sondern auch die Entwicklungsgeschichte verdankt, so suchte man bei tieferem Eindringen in den Bau auch die Entwicklungsgeschichte der Geschwülste festzustellen. Nun sind wir freilich bei der Erforschung der Entwicklungsgeschichte einer Geschwulst we. sentlich übler daran als bei der Erforschuug der Entwicklungsgeschichte eines normalen Organs. Jede Geschwulst ist wie ein Individuum nur einmal vorhanden, es sind fertige Zustande, die wir untersuchen müssen. Das ist bei dem Organ eines Säugetiors ähnlich; aber wir haben hier die Möglichkeit, beliebig viele Stadien der Entwicklung der Untersuchung zu un. terwerfen und eine den wirklichen Verhältnissen entsprechende Reihe aufzustellen. Wenn wir aber z. B. zwei Carcinome haben, vielleicht auch von derselben Stelle stammend, so ist es durchaus nicht ohne weiteres möglich, das kleinere etwa als ein Entwicklungsstadium des größeren aufzufassen.

Um die Histogenese einer Geschwulst zu begründen, die Entwicklung also auf ihren Anfang zurickzuführen, sind wir im wesentlichen auf den Vergleich verschiedener Stellen der Ge schwulst untereinander, sowie den Vergleich der Geschwulstzellen mit dem benachbarten Gewebe angewiesen. Dafl dieser Weg schwierig ist und leicht zu voreiligen Schlüssen führt, das weil jeder pathologische Anatom, das beweist die Geschichte unserer Wissenschaft. Nur der vergleichend-experlmentelle Weg vermag hier Aussichten auf eine bessere Begründung unserer Ansichten zu geben.

Seit die Entwicklungsgeschichte in moderner Weise be- 
trieben wurde, hat es nicht an Forschern gefehlt, denen die Beschreibung des Entwicklungsganges, die Erkenntnis der Veränderung der Form, der "formalen Genese" nicht genügte, die vielmehr sich auch die Frage vorlegten: Wodurch und warum kommt dieser Entwicklungsvorgang zustande, welches sind die äußeren und inneren Bedingungen, kurz, wie vollzieht sich die "kausale Genese"? Schon der Altmeister der Entwicklungsgeschichte, Karl Ernstv. Bär gab „Beobachtung und Reflexion“, und zwar tiefe, gründliche Reflexion auch über Fragen der kausalen Genese. Durch das Verdienst von Roux ist die kausale Forschung ein eigener Zweig der Entwicklungsgeschichte geworden, von Roux als Entwicklungsmechanik, von anderen, vor allem von Driesch als Entwicklungsphysiologie bezeichnet.

Auch in der Lehre von den Geschwülsten wurde die Frage nach der kausalen Genese zugleich mit der formalen Genese oder noch früher als diese behandelt. Häufig hielt man formale und kausale Genese nicht genügend auseinander. Die Geschwulstlehre, als ein Teil der Pathologie, hat die Frage nach dem Warum unter Zuhilfenahme sonstiger pathologischer Erfahrungen zu lösen gesucht. Die Erkennung der kausalen Genese oder Aetiologie war in dem letzten Drittel des 19. Jahrhunderts insbesondere für die Infektionskrankheiten mächtig getördert worden. Vollkommen berechtigterweise versuchte man, sich diese Erfahrungen auch für die Aetiologie der Geschwülste zunutze zu machen.

Wir können also in der Geschwulstforschung drei Gebiete, die natürlich sich eng berühren, einander ergänzen, unterscheiden und dementsprechend eine Einteilung treffen, wenn wir versuchen, einige wichtige neuere Erkenntnisse uns klar zu machen:

1. Morphologieder Geschwülste. 2. Formale Genese. 3. Kausale Genese.

Werfen wir kurz noch einen Blick auf die Methoden der Geschwulstforschung. Zunächst müssen wir die verschiedenen Methoden der Morphologie anwenden. Wir untersuchen möglichst viel und möglichst eingehend alle erreichbaren menschlichen Geschwülste, alle histologischen Untersuchungsmethoden müssen in zweckmäßiger, dem Einzelfall angepaßter Auswahl herangezogen werden. Die Vergleichung der menschlichen Geschwülste untereinander, sowie mit anderen menschlichen pathologischen Vorgängen und Gebilden hat bedeutende Förderung gegeben. Insbesondere die Mißbildungslehre, die Teratologie, ist für die Geschwulstlehre, die Onkologie von der allergrößten Bedeutung. Die Vergleichung mit tierischen Geschwülsten, die vergleichende Pathologie, verspricht, insbesondere vereint mit der experimentellen Methode, wertvolle Aufschltïsse.

Die experimentelle Methode endlich hat für viele Fragen der Geschwulstlehre, sowohl für formale wie kausale Genese die größte Bedeutung, und es ist vielleicht der größte Fortschritt der neuesten Zeit, daß wir gelernt haben, eine fruchtbare Methode experimenteller Uebertragung beim Tier anzuwenden.

Im folgenden soll es unsere Aufgabe sein, einige neuere Forschungen auf dem großen Gebiete der Geschwülste, die besondere Bedeutung erlangt haben, herauszuheben und so Anregung zu weiterer Beschäftigung mit der so interessanten Geschwulstbiologie zu verbreiten. Wir folgen der vorhin genannten Einteilung. ${ }^{1}$ )

Wenn auch die Morphologie der Geschwülste das am längsten und gründlichsten durchforschte Gebiet ist, so darf man doch nicht glauben, daf auf diesem Gebiet in den letzten Jahren keine wichtigen Entdeckungen mehr gemacht worden wären. Aus der Morphologie konnte vielfach ein histogenetischer Schluß gezogen werden, wie ja die formale Genese aufs engste mit der Morphologie zusammenhängt. Wir werden naturgemäß bei Besprechung der Morphologie einzelner Geschwulstarten sogleich auch die Histogenese dieser Geschwïlste behandeln müssen. Im zweiten Abschnitt werden wir uns

1) Ich empfehle für kurze Orientierung außer den Darstellungen in den Lehrund Handbuchern (namentlich Borst, Oeschwülste) die Monographie von v. Dungern und Werner, Das Wesen der bossartigen Geschwülste, sowie far die experImentelle Krebsforschung Insbesondere Dietrich, Der heutige Stand der experimentellen Krebsforschung, Deutsche medizinische Wochenschrift 1907 , No 13. mehr mit allgemeinen Theorien der formalen Genese als mit einzelnen Geschwulstarten beschäftigen.

Ich greife zunächst einige $\mathrm{B}$ e is piele heraus, die uns zeigen sollen, daß die morphologische Forschung zur Vertiefung und Verbreiterung unseres Wissens von den Geschwülsten geführt hat.

Wir haben gelernt, die hypernephroiden Tumoren als eine eigene Gattung von verwandten Goschwülsten abzugrenzen. Nicht allzuselten begegnet man auf der Nierenkapsel, mitunter in das Nierengewebe sich einsenkend, kleinen, gelblichen Knötchen, die oft linsenförmig abgeflacht sind. Die gelbe Farbe begünstigte die Auffassung dieser Tumoren als Fettgeschwülste. Heute bezeichnen wir sie als Nebennierenadenome. Wir wissen, daß das Charakteristische dieser Geschwülstchen der Aufbau aus Nebennierenzellen ist. Es ist während der Entwicklung versprengtes Nebennierengewebe, das am häufigsten an der Niere, gelegentlich auch an anderen Stellen, am Ligamentum latum etc. vorkommt. Nicht sehr häufig, immerhin aber auch nicht allzu selten, finden wir die Niere von einer Geschwulst zerstört, die, von gelblicher Farbe, große Ausdehnung erreichen kann und meist sehr reichlichen Zerfall erkennen läßt. Diese Geschwülste lassen als charakteristischen Bestandteil ebenfalls Nebennierengewebe erkennen, es sind die hypernephroiden Tumoren. Sie können sich durchaus verhalten wie bösartige Geschwülste, sie machen Metastasen und führen den Tod des Trägers der Geschwulst herbei. Wir sehen sie als eine eigene Gruppe von Geschwülsten an, die sich am engsten an die erwähnten Nebennierenadenome anschließt.

Ein weiteres Beispiel für die Fortschritte in der morphologischen Erkenntnis der Geschwïlste sind die Chorionepitheliome. Es ist Marchands Verdienst, die moderne Auffassung dieser Geschwïlste begründet zu haben. Nach Schwangerschaften, namentlich nach Blasenmole, kommen im Uterus weiche, sehr blutreiche Geschwülste vor, die eine sehr bedeutende Bösartigkeit aufweisen. Sie wurden früher unter dem Namen der Deciduome beschrieben. Heute geht die allgemeine Anschauung dahin, daß Zellen der Chorionzotten, die eine aus dem Syncytium und der Langhansschen Schicht bestehende Zellbekleidung besitzen, das charakteristische Element der genannten Tumoren bilden. Nachdem diese Auffassung allgemein geworden war, lernte man einige sehr interessante Vorkommnisse des Chorionepithelioms kennen. Man fand, daß primäre Chorionepitheliome entfernt vom Uterus, ja von den Genitalien überhaupt auftreten können. Es kann sich dabei nur um Geschwulstbildung aus verschleppten Chorionzotten handeln. Es ist das sicher eine wichtige Erkenntnis, die auch für manche andere Geschwülste zu denken gibt. So kommen Knochentumoren vor, die den Bau einer Struma colloides aufweisen. Man darf dem Gedanken Raum geben, dab von verschleppten Schilddrüsenzellen diese Geschwülste vielleicht ihren Ausgang nehmen könnten.

Besonders ist in letzter Zeit das Vorkommen von Chorionepitheliomen beim männlichen Geschlecht besprochen. Es handelt sich um das Vorhandensein von chorionepitheliomartigen Wucherungen in Teratomen, diese Wucherungen können aber diesen Geschwülsten durchaus das Gepräge geben. Es ist allerdings noch eine der Untersuchung werte Frage, ob nicht andere Geschwülste, vor allem solche, welche von den Endothelien ausgehen, in weitgehender Weise den Bau des Chorionepithelioms nachahmen können. In Heidelberg wurde ein später von Marx beschriebener Tumor gefunden, der in der Leber eines Mannes seinen Sitz hatte und einen dem Chorionepitheliom ähnlichen Bau aufwies. Solche Befunde sind von großer Wichtigkeit und sind zugleich geeignet, auch für einen Fortschritt in der Erkenntnis der von Endothelien abstammenden Geschwülste, der sogenannten Endotheliome zu wirken. Die Morphologie der Endotheliome ist noch keineswegs geklärt, und unsere besten Geschwulstkenner sind gerade im Punkt Endotheliome recht geteilter Meinung.

Einen Versuch, eine neue große Gruppe von bösartigen Geschwülsten abzugrenzen, hat vor nicht allzu langer Zeit Krompecher gemacht. Er bezeichnete als Basalzellenkrebs eine Reihe von Geschwülsten, die nach seiner Meinung eine 
Art Zwischenstellung zwischen Carcinom und Sarkom einnehmen. Die Geschwulstgruppe, von der er ausging, ist freilich eine altbekannte, als Retezellencarcinome, Matrixcarcinome u, a. bezeichnete Art des Hautkrebses. Gerade an seinen Basalzellenkrebsen versuchte Krompecher Zellverbindungen nachzuweisen, die zwischen Epithelzellen und Bindegewebszellen bestehen sollen. Eine Nachprüfung dieser Angaben mit besonders geeigneten Methoden ist jedenfalls eine lohnende Arbeit. Es kann keinem $\mathrm{Z}$ weifel unterliegen, daß eine Bestätigung oder Widerlegung der Krompecherschen Befunde in gleicher Weise von Wichtigkeit für die Morphologie der Geschwülste wäre.

Von Interesse sind auch die Untersuchungen über Melanome, Melanosarkome oder Chromatophorome, wie Ribbert diese Geschwülste genannt hat. Man weiß schon seit langer Zeit, daß namentlich im Auge, dann in der Haut, im Anschluß an Naevi pigmentierte, bösartige Geschwülste sich entwickeln können, die man früher allgemein zu den Sarkomen rechnete und als Melanosarkome bezeichnete. Neuerdings wurde die Stellung der von den Naevis abzuleitenden Geschwülste zweifelhaft, Unna u. a. wollten diese Tumoren zu den Carcinomen gestellt wissen (Melanocarcinoma). In neuester Zeit hat nun Ribbert diese Geschwülste als Chromatophorome beschrieben. Die Chromatophoren oder Pigmentzellen sind in ihrer Morphologie charakteristische Zellen, die zwar zur Bindegewebsgruppe gehören, aber darum doch eine solche Eigenart beanspruchen, daß sie als besondere Zellen angeführt werden dürfen. Ihre charakteristischen Merkmale treten namentlich bei frischer Untersuchung hervor. Sie zeigen bei derselben vielfache bandartige Ausläufer, sind reichlich verzweigt. Sie besitzen ein reichliches Protoplasma, was sie von anderen Bindegewebszellen unterscheidet. Sie allein vermögen Pigment zu bilden, obgleich sie wenigstens beim Menschen diese Fähigkeit keineswegs stets betätigen. Es gibt also auch unpigmentierte Chromatophoren. Beim Menschen sind die Chromatophoren, wie Ribbert schreibt, „nur an gewissen Körperstellen (After, Mammilla) regelmä日lig mit Pigment versehen, auf der übrigen Haut dagegen größtenteils oder alle frei von Farbstoff. Sie existieren hier aber nichtsdestoweniger und ëußern unter besonderen Bedingungen ihre pigmentbildende Fähigkeit. Das ist besonders in abnormen, kongenital umgebildeten Hautpartien der Fall" (Naevi). Ribbert behauptet nicht nur, daß die Chromatophoren die charakteristischen Bestandteile der Chromatophorome sind, er geht vielmehr so weit anzunehmen, daß die Chromatophorome nur aus einer Zellart, die sich allerdings in verschiedenen Zuständen und Entwicklungsstadien befindet, beständen. Es muß betont werden, daß durch Ribberts Untersuchungen der weiteren Forschung über die Morphologie der Melanome große Anregung gegeben ist. Es wird sich darum handeln, durch vergleichende Untersuchungen festzustellen, ob die Chromatophorome tatsächlich aus einer Zellart bestehen, oder ob diese Deutung widerlegt werden kann.

Nicht nur neue Gruppen von Geschwülsten waren es, die durch morphologische Untersuchungen der letzten Jahre festgestellt werden konnten, auch an wohldurchforschten Geschwülsten wurde manches Neue entdeckt, so vor allem am Carcinom. Das Dogma früherer Jahrzehnte, daß die Krebszellen keine Funktion, wenigstens keine der normalen ähnliche Funktion auszuüben vermöchten, ist widerlegt. Wir wissen heute, daß verhältnismäßig weitgehende Funktionen von Krebszellen ausgeübt werden können. Es läßt sich das namentlich an den Metastasen beobachten. Daß Metastasen von Darmklebsen Schleim produzieren, ist nichts Ungewöhnliches. Doch auch Galle kann z. B. von Lebercarcinomen oder deren Metastasen sezerniert werden, desgleichen Colloid von Schilddrüsencarcinomen, von primären sowohl wie metastatischen. $\mathrm{Ja}$, wie insbesondere v. Han semann dargetan hat, die Funktion innerhalb eines Carcinoms kann von Bedeutung für den Gesamtorganismus werden. Bei Carcinom der Nebennieren z. B. kommt es nicht zur Addisonschen Krankheit, weil die innere Sekretion der Nebennieren durch das Carcinom nicht aufgehoben wird.

Für sehr wichtig halte ich auch Resultate, die M. B. Sch midt in einer Schrift über Verbreitungswege des Carcinoms 1903 niederlegte In einer verhältnismäßig großen Zahl von Car. cinomfällen fand M. B. Schmidt Krebsembolien, die nur mikroskopisch nachweisbar waren, in den Lungengefäßen. Ist schon diese Tatsache an sich eine sehr interessante, so ist insbesondere ein Resultat für die Praxis sowohl wie für unsere Anschauungen vom Wesen des Carcinoms überhaupt von Wichtigkeit. Schmidt konnte zweifellos nachweisen, daß „die häufig in die kleinen Lungenarterien lebenskräftig eingeschwemmten Krebszellen in großem Umfang zugrunde gehen". Damit ist die prinzipielle Möglichkeit der Heilung von Carcinom auch für den Menschen dargetan, wofür ja einige bei Operationen gewonnene Erfahrungen schon sprechen. Die experimentelle Forschung hat die Rückbildung von bösartigen Tumoren bei Mäusen als keineswegs allzu seltenès Vorkommnis dargetan.

Recht bedeutungsvoll ist auch die Abgrenzung, welche die Lym phosark ome in der Abhandlung von Schmidt von anderen Tumoren erfahren. Daß die Lymphosarkome eine Sonderstellung unter den Tumoren einnehmen, ist eine Ueberzeugung, die wohl viele pathologische Anatomen aus ihrer Erfahrung gewonnen haben. Ich habe dieser Ueberzeugung in meinem Lehrbuch der Mißbildungen Ausdruck gegeben und bin der Meinung, daß möglicherweise die Lymphosarkome einmal ganz von den echten Geschwülsten geschieden werden müssen, wie es mit dem Tuberkel und dem Gumma gegangen ist. Die Anknüpfungen des Lymphosarkoms nach der Seite der Leukämie hin liegen klar. Jedenfalls können Uebertragungsversuche von Lymphosarkomen nicht ohne weiteres den Uebertragungsversuchen anderer Tumoren, von Carcinomen oder Sarkomen oder Chondromen an die Seite gestellt werden. Die Erforschung der Lymphosarkome, und namentlich der Wachstumsart der Lymphosarkome, gehört zu den weiteren morpho:logischen Aufgaben der Zukunft.

Wir wollen jetzt zu dem zweiten Abschnitt unserer Betrachtung übergehen und über die formale Genese der Tumoren einiges berichten.

Wie ich schon andeutete, ist die Erkenntnis, daß formale und kausale Genese auch für Geschwülste etwas Verschiedenes ist, das auseinander gehalten werden muß, noch keineswegs ein Allgemeingut aller Aerzte geworden. Die bedeutsamste Anregung haben unsere Vorstellungen von der formalen Genese durch die Cohnheimsche Theorie erhalten. Es ist in der Geschichte der Wissenschaft die Cohnheimsche Theorie ein ausgezeichnetes Beispiel dafür, wie sehr eine kühne Verallgemeinerung schon gefundener Einzeltatsachen zu einer umfassenden Theorie anregend und fördernd auf die Wissenschaften zu wirken vermag, selbst wenn in der Begründung noch zahlreiche Lücken nachgewiesen werden können. Anderseits zeigt uns auch gerade die Cohnhoimsche Theorie, daB eine zu weit gehende Anwendung, namentlich wenn die nötige Kritik vergessen wird, schädlich wirken kann und zu einem Rückschlag führen muß. Der Cohnheimschen Theorie wird das große Verdienst immer zuerkannt werden müssen, daß sie die Bedeutung der Entwicklungsstörung für die Entstehung der Geschwülste in helles Licht gesetzt hat. Wenn man auch schon früher abnorme Entwicklungsvorgänge für einzelne Geschwülste als bedeutungsvoll angesehen hatte, so ist doch erst durch die Cohnheimsche Theorie die Wichtigkeit der Entwicklungsstörungen voll gewürdigt worden.

Durch die neueren Untersuchungen ist diese hohe Bedeutung bestätigt worden. Es ist vor allem Ribberts Verdienst, unermüdlich an dem Ausbau der durch Cohnheim gegebenen Grundlagen in origineller Weise gearbeitet zu haben. Die "Ribbertsche'Theorie", die sich an die Cohnheimsche anschließt, ist allgemein bekannt. Eine Darstellung derselben ist nicht ganz leicht, da Ribbert häufig Aenderungen vorgenommen hat und noch in jüngster Zeit eine nicht unwesent. liche Modifikation für angezeigt hielt. Ich will auch im folgenden keineswegs die Ribbertsche Theorie im ganzen zur Darstellung bringen, vielmehr nur die Punkte herausheben, die für die formale Genese der Geschwülste von Bedeutung geworden sind. Als eine außerordentlich wichtige Förderung, die wir durch Ribberts Untersuchungen in erster Linie gewonnen haben, betrachte ich die Lehre von dem Wachstum der Geschwülste, 
Zwar ist Ribberts Ansicht durchaus noch nicht allgemein anerkannt; daß aber durch seine Behauptungen die Untersuchungen über das Wachstum der Geschwülste mächtig gefördert sind, wird jeder zugeben. Nach Ribbert findet das Wachstum einer jeden Geschwulst ausschließlich aus sich heraus statt, es konımt nicht vor, daß ein Magencarcinom zum Beispiel sich dadurch vergrößert, daß auch die umgebenden Magendrüsen carcinomatös werden. Diese können vielmehr von dem Carcinom verdrängt werden, aber nicht in die carcinomatöse Wucherung einbezogen werden. Am besten kann man sich das Wachstum einer Geschwulst an einer Metastase klar machen. In einer Lebermetastase eines Magencarcinoms wächst der Tumor nur aus sich heraus. Hier kann kein Zweifel an der Richtigkeit der Ribbertschen Behauptung sein. Auch scheinen die experimentellen Erfahrungen an Mäusetumoren - für Ribbert zu sprechen. Eine Ausnahme, welche die Lymphosarkome machen dürften, könnte eher zur Abtrennung der Lymphosarkome von den echten Geschwülsten als zur Gegnerschaft gegen die angeführte Ribbertsche Behauptung führen. Es läßt sich zurzeit noch nicht feststellen, ob der Satz: "Die Geschwülste wachsen nur aus sich heraus", allgemeine Gulltigkeit hat, doch hat sich, soweit ich die Literatur übersehe, diese Ribbertsche Feststellung immer mehr Anhänger erworben. Jedenfalls ist es eine Aufgabe, bei jeder Tumoruntersuchung sich die Frage vorzulegen, ob nach dem Ausfall der Untersuchung zugunsten oder zuungunsten des genannten Axioms etwas ausgesagt werden kann.

Ein zweiter von Ribbert aufgestellter Satz dürfte schwerer als der erste eine allgemeine Anerkennung finden. „Das Wachstum der Geschwülste vollzieht sich durch Fortfall von Wachstumswiderständen." Ein Beispiel für den Fortfall von Wachstumswiderständen kann in der Histogenese des Carcinoms gefunden werden. Das Epithel vermag erst zu wachsen, wenn der Wachstumswiderstand des Bindegewebes etwa durch eine Entzündung des Bindegewebes aufgehoben ist. Die kleinzellige Infiltration in der Umgebung vieler kleiner Carcinome ist von Ribbert in dem eben geschilderten Sinne verwendet worden. Es kann durch diese Entzündung zu einer postfötalen Ausschaltung von Epithel kommen und damit die Veranlassung zum Wachstum einer Geschwulst gegeben sein. Wir sehen also, daß auch postfötal erst sich die Entstehung eines Carcinoms anbahnen kann, im ganzen aber legt Ribbert sehr großen Wert auf die fötale Entwicklungsstörung. Die Bedeutung derselben für die Geschwulstentstehung ist namentlich durch die Untersuchungen von Wilms über die Mischgeschwülste beleuchtet worden. Dab die Mischgeschwülste eine Entwicklungsstörung zur Voraussetzung haben, eine "Keimausschaltung" in weiterem Sinn, dürfte jetzt wohl allgemein angenommen sein. Der Zusammenhang der Mischgeschwülste mit den Teratomen ist nach Wilms' Untersuchungen kaum in Abrede zu stellen, ebenso wenig, dab die Reihe: Doppelbidung, Teratom, Mischgeschwulst, einfache Geschwulst nicht nur eine äußerliche morphologische, sondern auch eine tiefere genetische Bedeutung hat.

Ich habe mich, von den Mißbildungen ausgehend, speziell um eine weitere Aufhellung dieses Zusammenhangs bemüht. Zunächst konnte ich es wahrscheinlich machen, dab die größere oder geringere Komplikation im Bau einer parasitären Doppelmißbildung mit der Entstehungszeit der Entwicklungsstörung in Beziehung steht. Je später die Entstehungszeit oder besser die teratogenetische Terminationsperiode angenommen werden muß. desto einfacher ist der Bau der parasitären Doppelbildung. Ein Epignathus. der völlige Organisation eines menschlichen Foetus erkennen läßt, ist auf eine frühere Entwicklungsstörung zurückzuführen als eine Mischgeschwulst des Gaumens. Der Begriff der teratogenetischen Terminationsperiode ist von einer Reihe von Forschern bereits anerkannt worden. Wie für die verschiedene Komplikation im Bau der Doppelbildungen die verschiedene teratogenetische Terminationsperiode in Anspruch genommen werden kann, ebenso kann der Unterschied von Doppelbildung und Teratom bzw. von sogen. bigerninalen und monogerminalen Teratomen auf eine verschiedene Terminationsperiode zurückgeführt werden, desgleichen der Unterschied von Teratom und Mischgeschwulst,
Man darf von einer onk ogenetischen Terminationsperiode in demselben Sinne reden wie von einer teratogenetischen. Daß die verschiedene onkogenetische Terminationsperiode bei $\mathrm{Ge}$ schwülsten, die auf eine Entwicklungsstörung bezogen werden dürfen, manche Verschiedenheit des Baues in richtiges Licht setzt, dafür kann z. B. die Arbeit Herx he imers über heterologe Cancroide als Beleg angeführt werden. Die dort beschriebenen Geschwülste dürften ihre spezielle Eigentümlichkeit der Entstehungszeit der Entwicklungsstörung, die in ihrer Genese anzunehmen ist, vielfach verdanken. Herxheimer kennt den Begriff der onkogenetischen Terminationsperiode nicht, doch sind mir seine Ausführungen besonders wertvoll, um die Frucht. barkeit dieses Begriffes darzutun.

Es liegt nicht in der Absicht dieses Vortrages, die Beziehungen von Mißbildungen, speziell Doppelbildungen, zu den Geschwülsten noch näher zu erläutern, die gegebenen Andeutungen müssen genügen. Das aber darf sicher behauptet werden, dab eine tiefere und gründlichere Erkenntnis der Mißbildungen auch der besseren Kenntnis der Geschwülste jedenfalls gute Dienste leisten wird. Schon die enge Verbindung, welche durch die Mißbildungen mit dem Gebiet der Entwicklungsgeschichte und Entwicklungsmechanik hergestellt wird, ist von Wichtigkeit.

Eine Aufklärung der Geschwiilste vom entwicklungs. mechanischen Standpunkt aus hat E. Albrecht versucht. Er betrachtet die Geschwülste als Organoide oder Organula und sieht in dieser Betrachtungsweise nicht nur ein Programm, sondern schon ein gut Teil der Lösung der Geschwulstfrage. Dieser optimistischen Auffassung kann ich nicht zustimmen, womit der Wert der Albrechtschen Forschungen und Darlegungen natürlich in keiner Weise herabgesetzt wird. Es ist für die Geschwulstlehre sicher von Wichtigkeit, die Tumoren nicht nur in einzelne Zellen zu zerlegen, sondern die Gesamtheit der an der Geschwulst beteiligten Gewebe in ihren $\mathrm{Be}$ ziehungen zueinander zu betrachten.

Gleichfalls für die formelle Genese der Geschwülste von Wichtigkeit waren die Ueberlegungen Benekes, der den $\mathrm{Be}$ griff der Kataplasie einführte, und $\mathrm{Hans} \theta \mathrm{manns}$, der lehrte, daß in der formellen Genese der Tumoren eine Anaplasie der Zellen zum Verständnis des Wachstums vorausgesetzt werden müßte. Die Ausführungen v. Hansemanns sind vielfach mißverstanden worden, haben sich aber bei richtiger Aulfassung dessen, was der Ausdruck "Anaplasie" bezweckt, als sehr fruchtbar erwiesen. Im wesentlichen dasselbe will $\mathrm{Haus} \theta \mathrm{r}$ ausdrücken, wenn er von neuen Zellrassen spricht, die beim Carcinom sich bilden sollen. Es stehen alle diese Anschauungen im Gegensatz zu der namentlich von Ribbert verfochtenen Idee, dab den Carcinomzellen keinerlei andere Qualitäten, namentlich in ihren Wachstumseigenschaften zukämen als den normalen Zellen. Es hat meines Erachtens die Erfahrung der experimentellen Onkologie sich der Anaplasie gunstig erwiesen; ich glaube nicht, daß wir ohne die Annahme auskommen, daß den Geschwulstzellen in mancher Beziehung andere Eigenschaften zugeschrieben werden müssen als den normalen Zellen. Auch hat Ribbert der Annahme der Anaplasie gewisse Konzessionen gemacht. Am besten geklärt ist vielleicht die formale Genese der Teratome. Für diese ist eine Entwicklungsstörung, wie wir sahen, unbedıngt anzunehmen. Es ist nun besonders interessant, daß die Teratome unter Umständen sich wie malıgne Geschwülste verhalten und Metastasen hervorbringen können.

Die Ergründung der kausalen Genese, der Aetiologie der Geschwülste, ist seit lange das Forschungsziel vieler Männer der Wissenschaft gewesen. Die Wege, die man wählte, waren je nach den Vorstellungen, die man sich über Möglichkeiten der Aetiologie bildete, verschieden.

Die Uebertragbarkeit namentlich des Krebses festzustellen, war das Streben der meisten Experimentatoren. Es ist bekannt, daß die Uebertragung von Mensch auf Tier nicht gelang, so oft sie auch versucht wurde. Dagegen war die Uebertragung von Tier auf Tier nach langen vergeblichen Versuchen erfolgreich. Es ist Pflicht, hier den Namen Hanaus zu nennen, der die ersten einwandireien Uebertragungen von Ratte zu Ratte vornahm. In neuerer Zeit sind Uebertragungen von Hundegeschwülsten, und namentlich von Mäusetumoren golungen. Es ist das Verdienst Jensens, die Uebertragung von Mäusetumoren 
in größerem Maßstabe unternommen zu haben. Jensen konnte hierbei wichtige Resultate fördern. Es haben dann $B$ ash ford und in Deutschland vor allem Ehrlich, unterstützt durch mehrere ausgezeichnete Mitarbeiter die Uebertragbarkeit der Tumoren studiert. Auch möchte ich den Namen des amerisanisehen Forschers Leo Loeb nennen, der nicht übergangen werden darf.

DaB zu den Vebertragungsversuchen von Tumoren mit Vorliebe Mäuse gewählt wurden, hat mancherlei Gründe. Zunächst wohl die verhältnismäßig leichte - wenigstens im Hin. blick auf andere Tiergeschwülste leichte - Uebertragbarkeit der Mäusetumoren, die allerdings durchaus nicht bei allen Stämmen in derselben Weise ausgebildet ist, wie wir sehen werden; gegenüber Hundeversuchen haben die Mäuseversuche jedenfalls den Vorteil bedeutend größerer Billigkeit, was bei dem großen Tiermaterial, das zu diesen Versuchen unbedingt notwendig ist, nicht unerheblich ins Gewicht fällt.

Der Prozentsatz, in dem die Mäusetumoren bei Uebertragungen "angehen", ist ein nach den Stämmen recht verschiedener. Die Schwankungen sind - falls überhaupt Erfolg erzielt wird - zwischen $10-90 \%$, ja einzelne Stämme können bis fast $100 \%$ Ausbeute geben. Bei Fortimpfung eines Tumors durch viele Generationen läßt sich durch künstliche Auslese, indem man nur die am schnellsten wachsenden Tumoren weiter impft, eine Steigerung der Uebertragbarkeit, der "Virulenz" des Tumors erzielen. Man kann so bis zu einem Angehen der Impfung in $100 \%$ der Fälle gelangen

Für die Morphologie der Tumoren besonders interessant 1st, daB ein Tumor bei wiederholten Uebertragungen seinen morphologischen Charakter ändern kann (Loeb, A polant). Im allgemeinen wird der Charakter der Geschwulst die meisten Mäusetumoren werden zu den Carcinomen gerechnet - zähe festgehalten, doch kommen, wie gesagt, Aenderungen vor. Eine gewisse Aenderung des Typus einer Geschwulst in der Metastase gegenüber. der primären Wucherung war in der menschlichen Pathologie wiederholt beobachtet worden. In dieser Richtung, d. h. in der Aenderung des Geschwulst. charakters am merkwürdigsten ist die Feststellung. welche von Ehrlich und Apolant gemacht wurde, daB ein Carcinom sich in ein Sarkom verwandeln kann. Auch Loeb verfügt über eine Beobachtung in gleichem Sinne. Ehrlich und Apolant fanden, dab die Transplantation eines Mäusecarcinoms lange Zeit fortgesetzt werden konnte, ohne Aenderung des Zell. charakters, daf dann aber (nach neun Monaten bis $21 / 2$ Jahren) in gewissen Fällen das Stroma der Carcinome sarkomatös werde. In den folgenden Generationen trat dann der Anteil des Carcinoms immer mehr zurück, bis er schließlich ganz zurückgedrängt und ein reines Spindelzellensarkom in die Erscheinung getreten war. - Es läßt diese Erfahrung jedenfalls die Frage von neuem aufwerfen, ob bei Carcinomübertragung in der Tat das Stroma stets aus dem Gewebe gebildet wird, in welches das Carcinom verpflanzt wurde, oder ob eine Uebertragung auch des Stromas wenigstens in gewissen Fällen vorliegt.

Daß nicht nur Tumoren von Mäusen und Ratten übertragbar sind, beweisen namentlich die Versuche Stickers unter Ehrlich am Hund. Freilich nimmt das Lymphosarkom, das er von Hund auf Hund übertrug, wie schon früher angedeutet, eine besondere Stellung unter den Geschwïlsten ein.

Durch die modernen Experimente sind wir nun in den Stand gesetzt, auch auf der Basis vorurteilsloser Versuche zu der parasitaren Theorie der Geschwülste Stellung zu nehmen. Ich kann es mir wohl ersparen, die einzelnen Parasiten zu erwähnen, die beschuldigt wurden, Carcinom hervorzubringen. Es nützte nichts, daß die pathologischen Anatomen immer wieder darauf verwiesen, dal die Geschwülste wohlbekannte Eigenschaften haben, die eine parasitäre Theorie sehr unwahrscheinlich machen Jedenfalls müßte ein Parasit gefunden werden, der gegenüber allen bisher bekannten andersartige, neue Eigenschaften aufweist. Daß das möglich ist, läßt sich natürlich nicht absolut in Abrede stellen, wahrscheinlich ist es nicht. Jedenfalls konnten die Pathologen wiederholt feststellen, daß alle bis jetzt beschriebenen Parasiten der Geschwülste einer ernsthaften Kritik nicht standhalten. Es ist eine schöne Bestätigung der pathologischen Kritik, wenn alle neueren her- vorragenden Experimentatoren sich auf den Standpunkt der pathologischen Anatomie gestellt haben. Die Redewendung, dadurch, daß die Krebszelle selbst als Parasit erkannt sei, wäre eine Aussöhnung des morphologischen Standpunktes mit dem parasitären zustande gekommen, halte ich für die Sache durchaus nicht fördernd. Es ist damit absolut nichts erklärt, und dieser Ausspruch ist nur geeignet, die vielen Probleme, die in der Morphologie und Biologie der Tumoren sich noch finden, zu verschleiern. Als eine Theorie kann ein solcher Ausspruch nicht gelten. Eine Theorie soll zum mindesten neue Fragestellungen anregen, oder sie soll zusammenfassend eine Reihe von Tatsachen unter einen Gesichtspunkt bringen. Das tut die eben genannte Theorie keineswegs. Ja, sie reißt sogar $\mathrm{Zu}$ sammengehöriges auseinander. Die Transplantation der Tumoren ist durchaus von dem Gesichtspunkt der normalen Transplantation zu verstehen. Das Verständnis dieser Tatsache wird durch die sogenannte Theorie, der wir oben gedachten, erschwert.

Das große Problem des Geschwulstwachstums läßt sich nur in Rücksicht auf die Erfahrungen des normalen Wachs. tums behandeln. Wir müssen versuchen, um mit J. Loeb zu reden, die Wachstumserscheinungen zu beherrschen. Wie kommt es, daß die Carcinomzelle frei und unabhängig von den Beziehungen und den regulierenden Einflüssen, die normalerweise jede Zelle an dem Platze des Organismus halten, an dem sie ihre Funktion auszuüben hat, in schrankenloser Weise sich vermehren kann? Wie schafft sich der werdende Tumor sofort die nötige Ernährung? Welche Korrelationen auf das Gefäßsystem und das Bindegewebe bedingen, daß sich hier ein nouer Gewebekomplex ausbilden kann? Kommen hier chemische oder osmotische Einflüsse ins Spiel? Durch welche Einflüsse können Wachtumspotenzen der Zellen, die wir ja aus der Regeneration kennen, angeregt werden? Reichen diese Wachstumspotenzen zur Erklärung des Geschwulstwachstums aus?

Alle diese und viele andere Fragen werden durch die "Theorie", die Krebszelle verhalte sich wie ein Parasit, nicht im geringsten gefördert. Nicht einmal die Erfahrung, daf man durch planmäBige Anwendung der Immunitätslehre, die ja in erster Linie auf die Erfahrungen der Bakteriologie aufgebaut ist, die Geschwulstlehre wesentlich förderte, berechtigt zu dem SchluB, daß die Theorie, die Krebszelle wirkt als Parasit, auch praktische Resultate gezeitigt habe. "Zellimmunisierung" war lange vor dieser Theorie bekannt, d. h. man wuBte, dab das Serum eines mit bestimmten Zellen behandelten Tieres spezifische Eigenschaften diesen Zellen gegenüber gewinnt.

Mit der Theorie des Parasitismus der Krebszelle kommen wir also keinen Schritt weiter als ohne dieselbe, und wenn diese Theorie nur dazu dienen soll, dem bisherigen Anhänger der parasitären Theorie der Geschwülste eine goldene Rückzugsbrücke zu bauen, so meine ich, dal wir dazu eine Theorie nicht nötig haben. Die Krebsforschung ist durch die parasitäre Theorie gefördert worden, einen wissenschaftlichen Irrtum frei anerkennen, ist noch nie als tadelnswert angesehen worden; oft hat ein großer Irrtum die Wissenschaft mehr gefördert als viele kleine Wahrheiten.

Daf gegen die parasitäre Theorie des Krebses verschiedene Tatsachen der experimentellen Forschung sprechen, sei jetzt noch kurz erläutert. Ehrlich konnte ein Chondrom ebenso übertragen wie Carcinom, und mit Recht bemerkte er, daß es für unser ganzes pathologisches Denken außerordentlich schwierig ist, die exzessive Wucherung eines Gewebes vom Typus des Knorpels auf einen Parasiten zurückzuführen 1). Noch mehr sprechen die Versuche dagegen, Tumoren mit nicht intaktem Zellmaterial zu erzeugen. Zerquetschen oder Zerreiben der Zellen genügt, um die Transplantation unmöglich zu machen, wie aus Versuchen Jensens, wie solchen von Loeb hervorgeht.

Für den Praktiker von größtem Interesse sind die Resultate über lmmunität, die namentlich von Ehrlich erzielt werden konnten. Ehrlich hat den Ausdruck "atreptische Immunitat" geprägt. Die Erklärung läßt sich leicht an folgenden Versuch anknüpfon. Ein Mäusetumor läßt sich auf die Ratte über-

1) A polant, im Handb. d. patholog Mikruurg. Ergănzunggb S 458, 
tragen und wächst hier zunächst mit ziemlicher Schnelligkeit weiter. Nach verhältnismäßig kurzer Zeit kommt aber das Wachstum zum Stillstand, und es findet eine völlige Rückbildung statt. Impft man zu der Zeit. zu welcher das Wachstum noch in Blüte stand, auf eine zweite Ratte über, so hat diese Transplantation keinen Erfolg, dagegen hat die Uebertragung auf eine Maus das Wachstum des Tumors in derselben zur Folge. Ehrlich nahm nun an, daf zum Wachstum der Mäusetumoren ein bestimmter Stoff $X$. der sich im Mäuseorganismus findet, nötig sei und daß dieser Stoff bei der ersten Uebertragung auf die Ratte, der er von Natur aus fehlt, mit übergeht, so dal das anfängliche Wachstum dos Tumors auf die Ratte sich erklärt. Die Unübertragbarkeit von der Ratte auf eine zweite Ratte, sowie die Rückbildung des auf die Raıte verimpften Mäusetumors würde mit dem natürlichen Fehlen des Stoffes X im Rattenorganismus in Zusammenhang stehen.

Fragen wir uns zum Schlul noch, ob die Therapie durch die experimentelle Forschung eine Förderung erfahren hat. Wir müssen uns gestehen, dab wir von praktisch brauchbaren Ergebnissen noch weit entfernt sind. Aber ein Hoffnungsstrahl ist uns durch die experimentelle Forschung Ehrlichs gezeigt worden, Wie erwähnt, konnte Ehrlich über hochvirulente Tumoren berichten, die in nahezu $100 \%$ der Ueberimpfungen angingen, während die Ausgangstumoren eine nur geringe Virulenz zeigten. Eine Impfung nun mit einem wenig virulenten Tumor, die keine Geschwulstbildung bewirkte, machte das geimpfte Tier gegen eine spätere Inokulation mit einem hochvirulenten Tumor unempfindlıch. Das ist wenigstens ein Fingerzeig für unsere therapeutischen Bestrebungen. Diese Experimente, sowie die Feststellung, daß kleine Krebsmassen auch beim Menschen durch die natürlichen Kräfte beseitigt werden können (vgl. die Untersuchungen von M. B. Schmidt u. a.), lehren uns, daß wir die Hoffnung auf endliches Gelingen einer Krebstherapie nicht aufgeben dürfen und daß wir weiter arbeiten sollen auf dem beschrittenen Wege. Jede, wenn auch kleine Förderung, ob auf experimentellem oder rein morphologischem Gebiete, soll uns willkommen sein; eine kritische Sichtung der gefundenen Tatsachen wird helfen, den Unterbau zu sichern, auf dem wir den Weiterbau fortführen wollen.

Znsammenfassung. Im vorliegenden Aufsatz sind einige neuere Resultate über Morphologie und Genese der Geschwälste erörtert. Es wurden als Beispiele der morphologischen Fortschritte die hypernephroiden Tumoren, Chorionepitheliome, Chromatophorome $\mathbf{u}$ a. angefuhrt. In dem Abschnitt über formale Genese wurden einzelne Teile der Ribbertschen Theórie erwànt, der Beziehungen der Mißbildungen zu den Geschwülsten, sowie der Anschauungen Albrechts, Hansemanns u. a. gedacht. Im letzten Abschnitt endlich sind einige Ergebnisse experimenteller Forschung besprochen. 